\title{
Effects of Celastrus paniculatus Willd. and Sida cordifolia Linn. in Kainic Acid Induced Hippocampus Damage in Rats
}

\author{
Gajanand Revanna Siddappa Pujari', Vijayalakshmi Subramanian', Suresh Rangogi Rao,* \\ 1'Department of Anatomy, Saveetha Institute of Medical and Technical Sciences, Poonamallee High Road, Chennai, Tamil Nadu, INDIA. \\ 2Department of Anatomy, Subbaiah Institute of Medical Sciences, NH-13, Purle, Shivamogga, Karnataka, INDIA.
}

\begin{abstract}
Aim: The purpose of this study was to behavioral and neuroprotective efficacy of Celastrus paniculatus Willd. and Sida cordifolia Linn on hippocampus of the brain in kainic acid induced neurodegenerative diseases. Materials and Methods: A total of 30 albino Wistar rats were randomly divided into five groups: Vehicle control, kainic acid, kainic acid + seed oil of Celastrus paniculatus (SOCP), kainic acid + aqueous root extract of Sida cordifolia (ARESC), kainic acid + SOCP + ARESC. The following was analyzed in all groups: (a) Behavioral tests- Hole board test, Shuttle box test, Morris Water Maze test. (b)Blood parameters- RBC, WBC, Haemoglobin, Platelet count. (c)Serum parametersserum uric acid, serum cortisol, serum cholinesterase. Results: The kainic acid induced Alzheimer rats has shown significant changes towards normal behaviors. Total time spent in the dark chamber, no of head dips, time spent in head dips, latency to head dips, Number of rearing, latency of entrance (Sec) to the platform. The RBC count, platelet count, $\mathrm{Hb}$ content has shown a significant increase and WBC count has shown a significant decrease. A significant decrease in the serum cortisol and cholinesterase also shown by the rats treated with SOCP and ARESC as a mark of neuroprotection. Conclusion: SOCP and ARESC could be possibly used as anti-neurodegenerative agents for protecting hippocampus damage caused by kainic acid. However, further studies confirming its potential are certainly warranted.
\end{abstract}

Key words: Water maze test, Hole board test, Shuttle box test, Neuroprotection, Celastrus paniculatus, Sida cordifolia.

\section{INTRODUCTION}

Kainic Acid (KA), a potent agonist to the $\quad \alpha$-amino-3-hydroxy-5-methyl-4isoxazolepropionic acid (AMPA)/kainate class of glutamate receptors, is 30 -fold more potent in neuro-toxicity than glutamate. In rodents, KA injection resulted in recurrent seizures, behavioral changes and subsequent degeneration of selective populations of neurons in the brain, which has been widely used as a model to study the mechanisms of neurodegenerative pathways induced by excitatory neurotransmitter. ${ }^{1}$ There are a number of biological mechanisms involved in the development of neurodegenerative disease. One of the major mechanisms of neuronal death in acute and chronic neurodegenerative disease such as Alzheimer's Disease (AD), Parkinson's Disease (PD), Huntington's Disease (HD), Temporal Lobe Epilepsy (TLE) and Amyotropic Lateral Sclerosis (ALS) is excitotoxicity. Glutamate is a major excitatory neurotransmitter which plays an important role in CNS. ${ }^{2}$ The abnormally hyperphosphorylated tau is the primary component of NFTs, which is positively correlated with the decline of memory and cognition in AD patients. Excitatory amino acids have been reported to play a significant role in the pathogenesis of AD. Kainic acid
Submission Date: 08-11-2018; Revision Date: 02-01-2019; Accepted Date: 22-04-2019

DOI: 10.5530/ijper.53.3.86 Correspondence: Prof. Suresh Rangogi Rao, Department of Anatomy, Subbaiah Institute of Medical Sciences, NH-13, Purle, Shivamogga, Karnataka, INDIA.

Phone: +919886000734 E-mail: s4chavan@yahoo. co.in

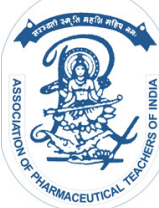

www.ijper.org 
(KA), an analog of glutamate, has been used to establish excitotoxicity models. Treatment with KA could induce tau hyperphosphorylation and neuronal death through activating GSK-3 $\beta$ and CDK5 pathway in both in-vitro and in-vivo models. ${ }^{3}$ Glutamate is a major excitatory neurotransmitter that mediates fast synaptic transmission and plays an important role in the mammalian brain and spinal cord. Excess glutamate is highly toxic to neurons. Glutamate acts through glutamate receptors. There are two major classes of glutamate receptors: ionotropic glutamate receptors (iGluR) and metabotropic glutamate receptors (mGluR). These receptors differed in terms of their functionality. iGluR mediate fast postsynaptic potentials by activating channels directly, while mGluR mediate slow postsynaptic potentials through $G$ protein activation. iGluRs can be divided into three types: N-methyl-D-apartic-acid (NMDA) receptors, $\alpha$-amino-3-hydroxy-5-methyl-4-isoxazole propionate (AMPA) receptors and kainite receptors. Kainic Acid (KA) exerts its neuroexcitatory property of binding to kainite receptors, which have presynaptic modulatory and post synaptic excitatory actions. KA activates glutamate receptors and the over activation of glutamate receptors produces neuronal membrane depolarization. This causes the influx of calcium ions and subsequently triggers excitotoxic neuronal death cascade events.

\section{MATERIALS AND METHODS}

\section{Requirements}

Surgical Scissors- Straight sharp/blunt $12 \mathrm{~cm}$, Narrow pattern forceps, Iris scissors, metal iron- free plate, small curved blunt forceps, large forceps with curved blunt to hold the brain, Graefe Forceps. RMS Semi-autoanalyser, Remi-centrifuge, Homogenizer, UV-Vis spectrophotometer, micropipettes. All chemicals, reagents, drugs, biokits used in this study were procured from authentic dealers.

\section{Collection of Plant and Preparation of Extract}

The roots of Sida cordifolia Linn were collected in and around the vicinity of Kerala. Seed oil of Celastrus paniculatus Willd was procured online. Identification and authentication was done by Taxonomist Regional Ayurveda Research Institute for Metabolic Disorders, Bengaluru, India. The plant roots were thoroughly washed with tap water to avoid dust and other unwanted materials accumulated on the roots from their natural environment. The dried roots were powdered by using an electric blender and the extraction was done in succession using Soxhlet extraction method. All the extracts were made of solvent free and concentrated. ${ }^{4}$

\section{Animals and Experimental Protocol}

Thirty albino Wistar rats (180-200g) were purchased from authenticated supplier Adita Biosys Private Limited, Tumakuru, Karnataka and were maintained in the animal house of PES College of Pharmacy, Bengaluru. All the animals were acclimatized for 10 days under standard husbandry conditions, i.e. the animals were housed in polypropylene cages maintained under controlled temperature at $23^{\circ} \mathrm{C} \pm 2^{\circ} \mathrm{C}$, relative humidity $45-55 \%$ and with $12 \mathrm{hrs}$ light:12hrs dark cycle, temperature and humidity was recorded daily using thermometer and hydrometer mounted in animal house. The animals had free access to standard rat pellet along with water supplied ad libitum under strict hygienic conditions. Each experimental group had a separate set of animals and care was taken to ensure that animals used for one response were not employed elsewhere. Animals were habituated to laboratory conditions for $48 \mathrm{~h}$ prior to an experimental protocol to minimize if any of non-specific stress. The experimental protocols were approved by the Institutional Animal Ethics Committee (IAEC) approval No- PESCP/IAEC/62/2016, Dated: 14/2/2017) and conducted according to CPCSEA guidelines, Govt. of India.

\section{Experimental design}

\section{Animal Grouping}

Animals were divided into five groups of six rats in each group. Group I (Vehicle control) received 0.5\% CMC throughout the course till 14 days. Group II received single dose of kainic acid $\left(25 \mathrm{mg} / \mathrm{kg}\right.$ b.w) i.p on $14^{\text {th }}$ day. Group III received SOCP $(500 \mathrm{mg} / \mathrm{kg}$ b.w) i.p for 14 days + kainic acid on $14^{\text {th }}$ day. Group IV received ARESC (250mg/kg b.w) p.o for 14 days + kainic acid on $14^{\text {th }}$ day. Group V received SOCP + ARESE + kainic acid.

\section{Estimation of Behavioral Parameters}

\section{Hole Board Test}

The Hole Board Test (HBT) apparatus is an area with walls around it to prevent escape. The floor of the area is covered with holes. Head dipping is commonly defined as when the animal puts his head into the hole until its ears are level with the floor or in modern devices when it breaks the infrared beam. Rearing is when the animal stands on its hind legs. Locomotion is the animal moving to a different area with all four paws. If the animal moves into the central area it is as separate observation because the central area is the most threatening to animals. These three behaviors are exploratory and the more they occur, the less anxious the animal is. On the 
other hand, if the animal doesn't show these behaviors then it is more anxious. The three common activities are head dipping, rearing and locomotion. ${ }^{5}$

\section{Shuttle Box Test}

The apparatus (Shuttle box) consisted of equal sized light and dark compartments $(20 \times 80 \times 20 \mathrm{~cm})$. This test has two stages, instruction and memory test. Prior to the experiment, all animals were placed into the apparatus for approximately $30 \mathrm{~min}$ (Habituation stage). Afterward, each rat was individually placed in the bright chamber and the door was opened. As soon as the rat entered the dark chamber, the door was closed and an electric shock was delivered by an isolated stimulator $(1 \mathrm{~Hz}, 3 \mathrm{sec}, 1 / 5 \mathrm{MA})$ through the grid floor. $24 \mathrm{~h}$ later, each rat was placed in the bright chamber for the retention test. The total time spent in The Dark Chamber (TDC) was measured. ${ }^{6}$

\section{The Morris Water Maze}

It is a widely used behavioral task in neuroscience for studying spatial learning and memory. This test is based on the fact that an animal will try to escape a stressful situation or stimulus, which in this case is a pool of water. The apparatus was prepared by placing the escape platform in the pool and filling the pool with clean, room temperature water to a depth where the platform in $1 \mathrm{~cm}$ above the water surface, approximately $40 \mathrm{~cm}$. Rats were allowed to swim and to search for the platform within $60 \mathrm{sec}$. It is common for animals to swim around the edge of the pool looking for an escape. After the completion of the testing trials, performed a probe trial for each animal in which the escape platform is removed. Recorded the number of times the animal crosses the platform location during thirty seconds. ${ }^{7}$

\section{Estimation of Blood Parameters Blood collection by Retro-Orbital technique}

The blood was withdrawn from the individual animals of all the 4 groups by retro-orbital puncture under light ketamine anesthesia ( $40 \mathrm{mg} / \mathrm{kg}$ i.p). The capillary is inserted into the medial canthus of the eye at about 30-degree angle to the nose. Slight pressure is applied along with rotation is applied to puncture the tissue and enter the plexus/sinus. The blood then enters through the capillary tube. The blood was collected in eppendorfs tube. Portion of the collected blood was used for the estimation of blood parameters- RBC, WBC, Haemoglobin, Platelet count.

The remaining blood was processed to serum and used for the estimation of serum parameters.
The blood sample which was collected in the eppendorfs tube did not contain any anticoagulant (Since serum was required for the estimation but not plasma) was kept in an upright position for approximately 30-40 min to facilitate clotting. The content was centrifuged at $3000 \mathrm{rpm}$ for $15 \mathrm{~min}$. The supernatant serum was transferred into another eppendorfs tube. The clear serum was used for the estimation of various serum biochemical parameters- uric acid, cortisol and cholinesterase.

\section{Estimation of Serum Uric Acid- Principle}

It is based on Trinder reaction. Uric acid is oxidized to allantoin by uricase with the production of $\mathrm{H}_{2} \mathrm{O}_{2}$. The peroxide reacts with 4-aminoantipyrine (4-AAP) and TOOS in the presence of peroxidase to yield a quinoneimine dye. The absorbance of this dye at $546 \mathrm{~nm}$ is proportional to uric acid concentration in the sample.

\section{Procedure}

Set up test tubes labeled Blank, Standard, Samples. Using micropipette transferred $20 \mu \mathrm{L}$ blank, standard and samples to appropriately labeled tubes. $1000 \mu \mathrm{L}$ working reagent was added to all the tubes, tap lightly and mixed well. The resultant mixture was incubated for $30 \mathrm{~min}$ at room temperature and absorbance and the serum uric acid concentration $(\mathrm{mg} / \mathrm{dl})$ was read at 590 $\mathrm{nm}(510 \mathrm{~nm}-630 \mathrm{~nm})$ using semi auto analyzer. ${ }^{8}$

\section{Estimation of Serum Cortisol}

The principle of the following enzyme immunoassay test follows the typical competitive binding scenario. Competition occurs between an unlabeled antigen (Present in calibrators, control and patient samples) and an enzyme-labeled antigen (Conjugate) for a limited number of antibody binding sites on the micro well plate. The washing and decanting procedures remove unbound materials. After the washing step, the enzyme substrate is added. The enzymatic reaction is terminated by addition of the stopping solution. The absorbance is measured on a microtiter plate reader. The intensity of the color formed is inversely proportional to the concentration of cortisol in the sample. ${ }^{9}$

\section{Estimation of Serum Cholinesterase}

Cholinesterases catalyses the hydrolysis of butyrylthiocholine, forming butyrate and thiocholine. Thiocholine reduces yellow potassium hexacyanoferrate (III) to colorless potassium hexacyanoferrate (II). The decrease in absorbance at $405 \mathrm{~nm}$ is proportional to the activity of CHE in the sample. ${ }^{10}$

\section{Statistical Analysis}


All the values was expressed as mean \pm SEM. Statistical comparisons were performed by one way Analysis of Variance (ANOVA) followed by Dunnett compare all columns versus control column using Graph Pad Prism version 5.0. ${ }^{*} P<0.05,{ }^{* *} P<0.01,{ }^{* * *} P<0.001$ was considered as significant compared to disease control.

\section{RESULTS}

\section{Shuttle Box Test}

Passive avoidance test results showed that TDC was increased in the kainic acid induced ND rats (II group), indicating memory impairment, than in the normal control group $(P<0.05)$. TDC was significantly decreased in the rats of group III, IV and V treated with KA acid and SOCP/ARESC when compared to group II (Table 1 and Graph 1).

\section{Hole Board Test}

The kainic acid induced ND rats (Group II) have shown a significant decrease in the No of head dips and latency to head dips (in sec) when compared to the normal control group (Group I). Group III and IV rats have shown a significant increase in the No of head dips and latency of head dips when compared to group II. Rats of group III, IV and V also shown significant increase in the latency to head dips (in sec) when compared to the group II (Table 2 and Graph 2).

The kainic acid induced ND rats (Group II) have shown a significant increase in the time spent in head dips and number of rearing (in sec) when compared to the nor-

\begin{tabular}{|c|c|c|}
\hline \multicolumn{3}{|c|}{$\begin{array}{c}\text { Table 1: Effects on total time spent in the dark cham- } \\
\text { ber (TDC) in normal and treated ND rats. }\end{array}$} \\
\hline Gpn=6 & Gp Name & TDC in sec \\
\hline I & Normal vehicle & $40.33 \pm 3.44$ \\
\hline II & ND rats & $312.5 \pm 5.19^{* * *} \mathrm{a}$ \\
\hline III & ND rats + SOCP & $46 \pm 7.97^{* * * b}$ \\
\hline IV & ND rats + ARESC & $42.1 \pm 6.20^{* * * b}$ \\
\hline V & ND rats + SOCP+ ARESC & $27.5 \pm 1.87^{* * * b}$ \\
\hline
\end{tabular}

mal control group (Group I). The rats in group III, IV and $\mathrm{V}$ have showed significant decrease in time spent in head dips when compared to group II rats. No significant difference observed in the number of rearing response when compared to group II rats (Table 2 and Graph 2).

\section{Morris Water Maze Test}

The kainic acid induced ND rats (Group II) have shown a significant difference in the latency of entrance (in sec) to the platform when compared to the normal vehicle group (Group I). SOCP/ARESC treated ND rats have shown a significant decrease in the latency of entrance (in sec) to the platform when the results are compared to the group II control rats (Table 3).

\section{Hematological Parameters}

The kainic acid induced ND rats have shown a significant decrease in the RBC count (Graph 3), platelet count (Graph 4) and (Graph 5) Hb content when compared to the normal vehicle group. Rats of groups III, IV and $\mathrm{V}$ have shown significant increase in the platelet count and $\mathrm{Hb}$ content when compared to control ND rats (gp II). However, rats in group III and IV have shown a significant increase in the RBC count when compared to control ND rats. The ND control rats have shown a significant increase in the WBC count when compared to normal vehicle group. Group III, IV and V rats have shown a significant decrease in the WBC (Graph 6) count when compared to control ND rats (II) (Table 4).

\begin{tabular}{|c|c|c|}
\hline Gpn=6 & Gp Name & $\begin{array}{c}\text { Latency of entrance }(\mathrm{sec}) \\
\text { to the platform }\end{array}$ \\
\hline 1 & Normal vehicle & $6.4 \pm 1.15$ \\
\hline II & ND rats & $60.66 \pm 2.52^{* \star * a}$ \\
\hline III & $\mathrm{ND}$ rats + SOCP & $25.16 \pm 1.65^{\star \star \star} b$ \\
\hline IV & ND rats + ARESC & $5.5 \pm 0.67^{* \star *} b$ \\
\hline $\mathrm{V}$ & $\begin{array}{c}\text { ND rats + SOCP+ } \\
\text { ARESC }\end{array}$ & $4.66 \pm 0.54^{* * * b}$ \\
\hline
\end{tabular}

\begin{tabular}{|c|c|c|c|c|c|}
\hline $\begin{array}{c}\text { Gp } \\
n=6\end{array}$ & Gp Name & No of head Dips & Time spent -sec & $\begin{array}{l}\text { Latency to head } \\
\text { dips (sec) }\end{array}$ & No of rearing \\
\hline 1 & Normal vehicle & $17.16 \pm 1.36$ & $3.83 \pm 0.65$ & $70.5 \pm 3.41$ & $7.33 \pm 0.67$ \\
\hline II & ND rats & $8.66 \pm 0.96^{* * * a}$ & $11.83 \pm 1.30^{* * * a}$ & $34.3 \pm 16.26^{* * * a}$ & $14.66 \pm 1.22^{* * * a}$ \\
\hline III & ND rats + SOCP & $13.33 \pm 0.92^{* * b}$ & $6.00 \pm 0.56^{* * * b}$ & $221.5 \pm 11.8^{* * * b}$ & $8.16 \pm 0.77^{*+* b}$ \\
\hline IV & ND rats + ARESC & $10.66 \pm 0.73$ & $5.16 \pm 0.52^{* * * b}$ & $146 \pm 9.11^{* \star \star b}$ & $7 \pm 0.63^{* * * b}$ \\
\hline V & $\mathrm{ND}$ rats + SOCP+ARESC & $15 \pm 0.63^{* *+* b}$ & $5.5 \pm 0.83^{* * * b}$ & $74 \pm 4.20^{* * \mathrm{~b}}$ & $5.66 \pm 0.54^{* * * b}$ \\
\hline
\end{tabular}




\section{Results of Serum parameters}

ND rats have shown decrease in the serum uric acid (Graph 7) levels and a significant increase in the serum cortisol (Graph 8) and serum cholinesterase (Graph 9) levels when compared to normal vehicle group (I). The group III, IV and V ND rats have shown a significant increase in the serum uric acid level and a significant decrease in the serum cortisol and serum cholinesterase levels when compared to control ND rats (group II) (Table 5).

\section{DISCUSSION}

In the shuttle box test, the control rats showed wellexpressed learning ability that was preserved during the memory retention test, suggesting the formation of memory traces. Animals treated with kainic acid showed poor learning and impaired memory paradigm.

The results at the behavioral level (Passive avoidance test) emphasize the role of SOCP/ARESC in the prevention of learning and memory impairments in kainic acid induced ND rats. These neuron protective effects may be due to the antioxidant and anti-inflammatory effects of SOCP/ARESC.

The kainic acid induced ND rats (Group II) have shown a significant decrease in the No of head dips and latency to head dips (in sec) when compared to the normal control group (Group I). Group III and IV rats have shown a significant increase in the No of head dips and latency to head dips when compared to group II. Rats in group III, IV and V also showed a significant increase in the latency to head dips (in sec) when compared to group II.

The kainic acid induced ND rats (Group II) have shown a significant increase in the time spent in head dips and number of rearing (in sec) when compared to the normal control group (Group I). The rats in group III, IV and V have shown a significant decrease in time spent in head dips when compared to group II rats. No significant difference observed in the number of rearing response when compared to group II rats.

Significant decrease in the serum uric acid content shown by the kainic acid induced ND rats (Group II) when compared to normal vehicle group rats (Group I). SOCP, ARESC and SOCP + ARESC treated ND rats (Group III, IV and V) have shown a significant increase in the serum uric acid level when compared to group II rats.

It was reported that high serum uric acid levels were significantly associated with decreased risk of Alzheimer disease. There is an inverse association between serum uric acid levels and Alzheimer's disease. High serum uric acid is a protective factor of Alzheimer's disease. SUA could exert neuro-protective effects against AD via its antioxidant capacities. It was reported that uric acid improves behavioral performances and cognition of rodents, increased TH-positive dopaminergic neurons and decreased GFAP- positive astrocytes in substantia nigra. Uric acid increased $\mathrm{mRNA}$ and protein expressions of $\mathrm{Nrf2}$ and three Nrf2-responsive genes, including gamma- glutamate-cystein ligase catalytic subunit. Uric acid significantly increased Superoxide Dismutase (SOD), CAT, Glutathione (GSH) levels and decreased Malondialdehyde (MDA) level in SN

Table 4: Effects on Hematological Parameters in Normal and Treated Rats.

\begin{tabular}{|c|c|c|c|c|c|}
\hline Gp $n=6$ & Gp Name & $\begin{array}{c}\text { RBC count } \\
\left(\times 10^{6} \mu \mathrm{l}\right)\end{array}$ & $\begin{array}{c}\text { WBC count } \\
\left(\times 10^{3} \mu \mathrm{l}\right)\end{array}$ & $\begin{array}{l}\text { Platelet count } \\
\qquad\left(\times 10^{4} \mu \mathrm{l}\right)\end{array}$ & $\begin{array}{c}\text { Hemoglobin } \\
\text { g/dl }\end{array}$ \\
\hline 1 & Normal vehicle & $8.45 \pm 0.56$ & $6.41 \pm 0.23$ & $2.5 \pm 0.25$ & $12.5 \pm 0.25$ \\
\hline II & ND rats & $3.97 \pm 0.28^{* *+a}$ & $14.51 \pm 1.71^{* * * a}$ & $1.9 \pm 0.20^{\star \star *} a$ & $9.5 \pm 0.23^{\star \star *} a$ \\
\hline III & ND rats + SOCP & $6.22 \pm 0.37^{* \mathrm{bb}}$ & $10.04 \pm 0.25^{\star * *} b$ & $2.2 \pm 0.19^{* * *} b$ & $12 \pm 0.22^{* * * b}$ \\
\hline IV & ND rats + ARESC & $5.31 \pm 0.55$ & $9.10 \pm 0.42^{* * * \mathrm{~b}}$ & $2.3 \pm 0.23^{* * *} \mathrm{~b}$ & $12.5 \pm 0.21^{* * *} b$ \\
\hline V & ND rats + SOCP+ RESC & $7.56 \pm 0.58^{* * * b}$ & $5.43 \pm 0.64^{* * *} b$ & $2.4 \pm 0.17^{\star * *} b$ & $12 \pm 0.19^{* * *} b$ \\
\hline
\end{tabular}

\begin{tabular}{|c|c|c|c|c|}
\hline Gp $n=6$ & Gp Name & Uric acid mg/dl & Cortisol $\mu g / d l$ & Cholinesterase U/L \\
\hline I & Normal vehicle & $2.51 \pm 0.008$ & 0.75 & 592 \\
\hline II & ND rats & $0.25 \pm 0.002^{* * * a}$ & $1.15^{\star * *} a$ & $788^{* * *} a$ \\
\hline III & $\mathrm{ND}$ rats + SOCP & $1.03 \pm 0.004^{* * x b}$ & $0.21^{* * *} \mathrm{~b}$ & $657^{\star \star *} \mathrm{~b}$ \\
\hline IV & ND rats + ARESC & $1.53 \pm 0.002^{* * * b}$ & $0.94^{* * *} \mathrm{~b}$ & $438^{\star * *} b$ \\
\hline $\mathrm{V}$ & $\begin{array}{c}\text { ND rats }+ \text { SOCP+ } \\
\text { ARESC }\end{array}$ & $2.05 \pm 0.004^{* * * b}$ & $0.95^{\star \star \star} \mathrm{b}$ & $401^{* * * b}$ \\
\hline
\end{tabular}




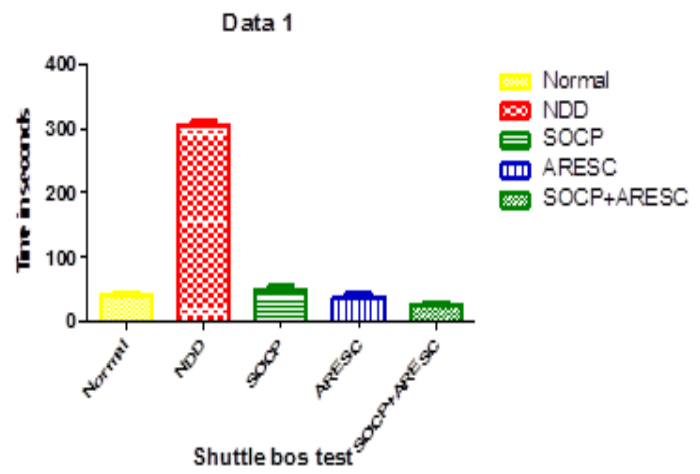

Graph 1: Showing effects on total time spent in the dark chamber (TDC) in normal and treated ND rats

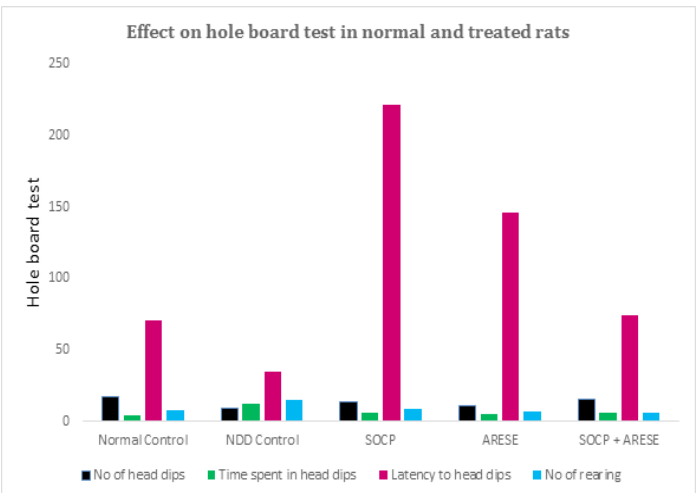

Graph 2: Showing effects on parameters of hole board test in normal and treated ND rats.

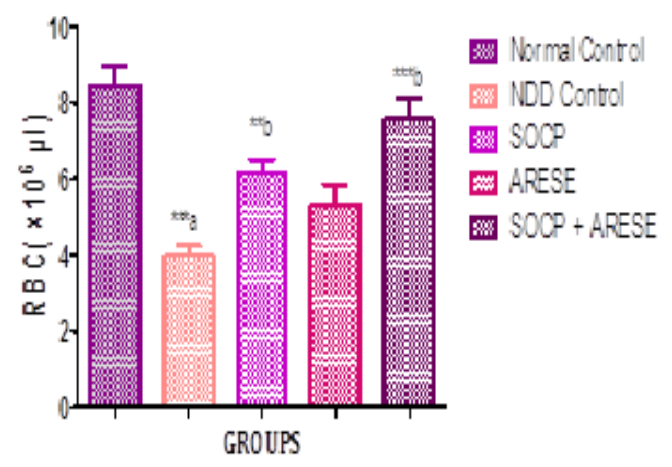

Graph 3: Showing effects on RBC count in normal and treated rats.

regions of MPTP-treated rodent. Uric acid inhibited the hippocampal expression of IL-1 $\beta$ and decreased serum and hippocampus levels of Interleukin-1 $1 \beta$ (IL-1 $\beta$ ), IL-6 and Tumor Necrosis Factor- $\alpha(\mathrm{TNF}-\alpha)$. The rats treated with ARESC and SOCP have shown significant increase in the serum uric acid levels in ND rats when compared to ND control rats. This reveals the protective role of ARESC and SOCP in preventing the kainic acid induced Alzheimer's disease. This neuro-protection may produce through modulation of neuro-inflammation and oxidative stress. ${ }^{11}$
Data 1

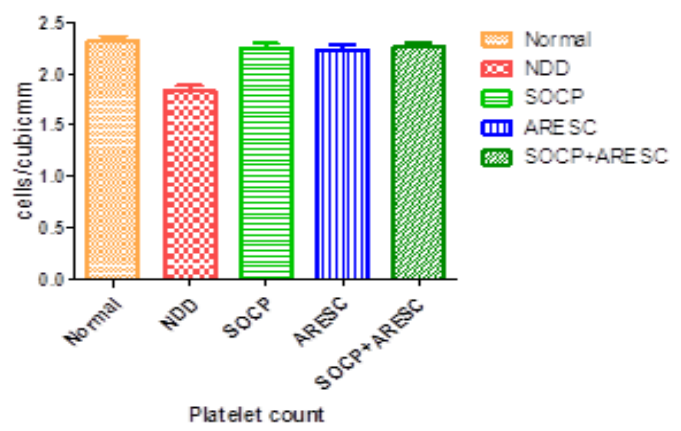

Graph 4: Showing effects on platelet count in normal and treated rats.

Da ta 1

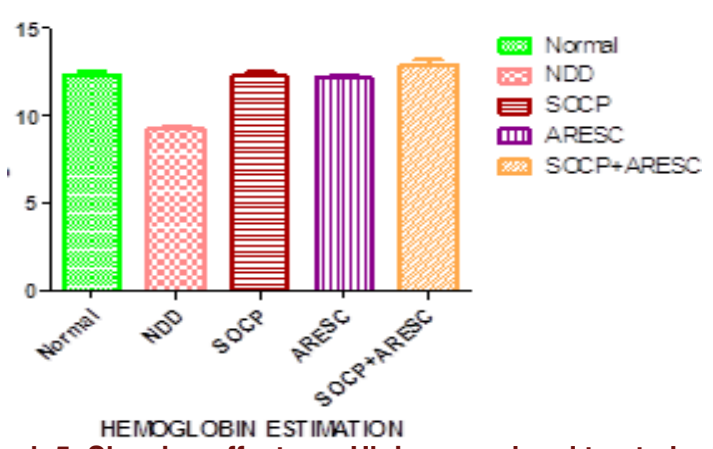

Graph 5: Showing effects on $\mathrm{Hb}$ in normal and treated rats.

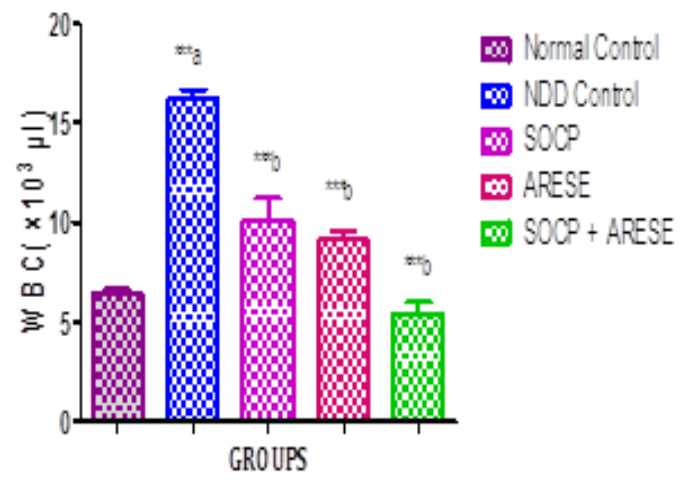

Graph 6: Showing effects on WBC in normal and treated rats.

\section{CONCLUSION}

This preliminary analysis tested the most promising behavioral like Shuttle box test, Hole board test, Morris water maze test, hematological parameters (RBC, WBC, platelet counts, $\mathrm{Hb}$ content) and serum markers- uric acid, cortisol and cholinesterase for Alzheimer's disease. It seems that SOCP and ARESC improved behavioral, blood and serum markers in a rat model of Alzheimer's disease. Thus, SOCP and ARESC could be possibly used as anti-neurodegenerative agents for protecting 


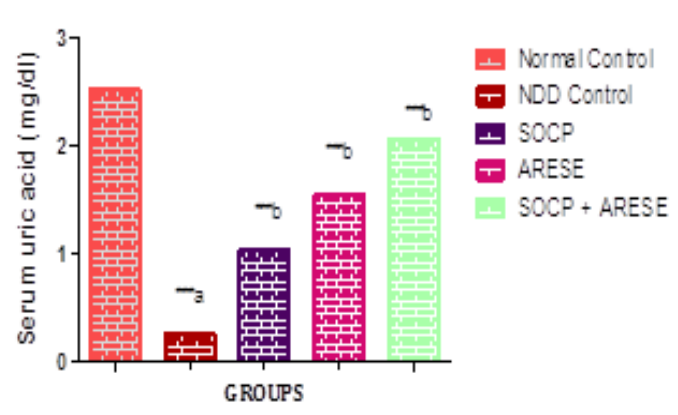

Data 1

Graph 7: Showing effects on serum uric acid in normal and treated ND rats.

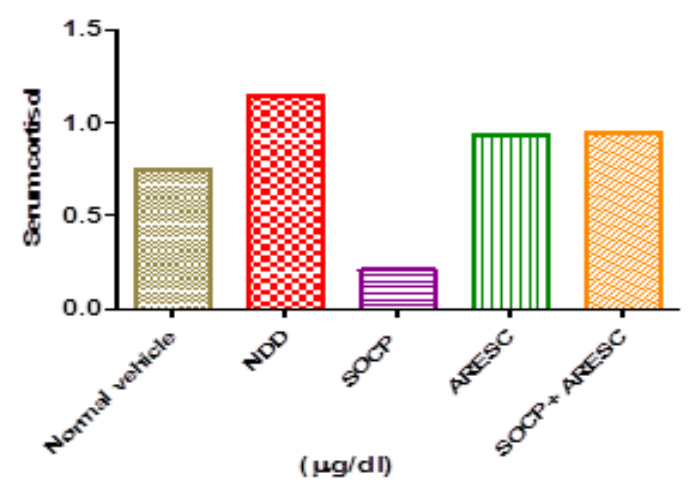

Graph 8: Showing effects on serum cortisol in normal and treated ND rats.

hippocampus damage caused by kainic acid. However, further studies confirming its potential are certainly warranted.

\section{CONFLICT OF INTEREST}

The authors declare no conflicts of interest.

\section{ABBREVIATIONS}

SOCP: Seed Oil of Celastrus paniculatus; ARESC: Aqueous root extract of Sida cordifolia; KA: Kainic Acid; ND: Neurodegenerative disease; AD: Alzheimer's disease; HBT: Hole board test; TDC: Dark Chamber; RBC: Red blood cell; WBC: White Blood Cell; Hb: Haemoglobin.
Data 1

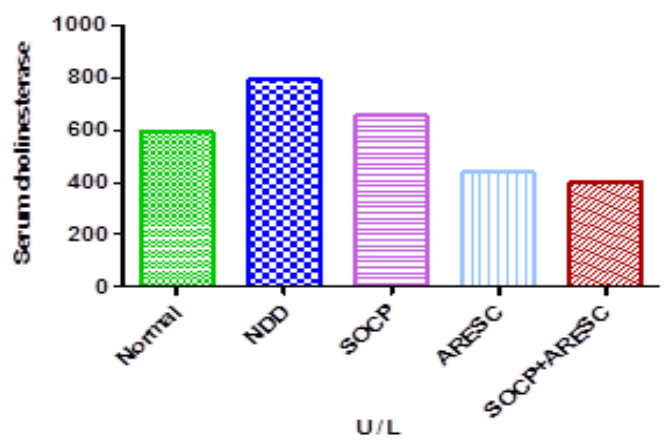

Graph 9: Showing effects on serum cholinesterase in normal and treated ND

\section{REFERENCES}

1. Xing-Mei Z, Jie Z. Kainic acid-induced neurotoxicity: Targetting glial responses and glial responses and Glia- derived cytokines. Curr Neuropharmacol. 2011;9(2):388-98.

2. Sumanth M, Mustafa SS. Antistress adaptogenic activity of Sida cordifolia roots in mice. Indian J Pharm Sci. 2009;7(3):323-4.

3. Cai S, Jia Z, Zixi L, Qingjie C, Weijian H, Liangtao X, et al. Melatonin mitigates kainic acid-induced neuronal tau hyperphosphorylation and memory deficits through alleviating ER Stress. Front Mol Neurosci. 2018;11(5):1-15.

4. Redfern J, Kinninmonth M, Burdass D, Verran J. Using Soxhlet ethanol to produce and test plant material (essential oils) for their antimicrobial properties. Journal of Microbiology and Biology. 2014;15(1):45-6.

5. Brown GR, Christopher N. The exploratory behavior of rats in hole board apparatus: Is head dipping a valid measure of neophilia?. Behav Processes. 2008;78(3):442-8.

6. Saba S, Mehrad J, Ali M, Nasrin SA. Effect of Scopolamine on avoidance memory and hippocampal neurons in male wistar rats. Basic and Clinical Neuroscience. 2011;3(1):9-15.

7. Angelucci MEM, Cesário C, Hiroi RH, Rosalen PL, Da Cunha C. Effects of caffeine on learning and memory in rats tested in the Morris water maze. Braz J Med Biol Res. 2002;35(10):1201-8.

8. Katsiki N, Papanas N, Fonseca VA, Maltezos E, Mikhalidis DP. Uric acid and diabetes: Is there a link?. Curr Pharm Des. 2013;19(27):4930-7.

9. Simon ML, Yen YL, Sophie JB, Tenielle P, James D, David A, et al. Plasma cortisol, brain amyloid- $\beta$ and cognitive decline in preclinical Alzheimer's disease: A 6-year prospective cohort study. Biological Psychiatry. 2017;2(1):45-52.

10. Gohar M, Nigel HG, Jalaluddin AK, Mohammad AK. Status of acetylcholinesterase and butyrylcholinesterase in Alzheimer's disease and type 2 diabetes mellitus. CNS and Neurological Disorders - Drug Targets. 2014;13(8):143-9.

11. Huang TT, Hao DL, Wu BN, Mao LL, Zhang J. Uric acid demonstrates neuroprotective effect on Parkinson's disease mice through Nrf2-ARE signaling pathway. Biochem Biophys Res Commun. 2017;493(4):1443-9. 


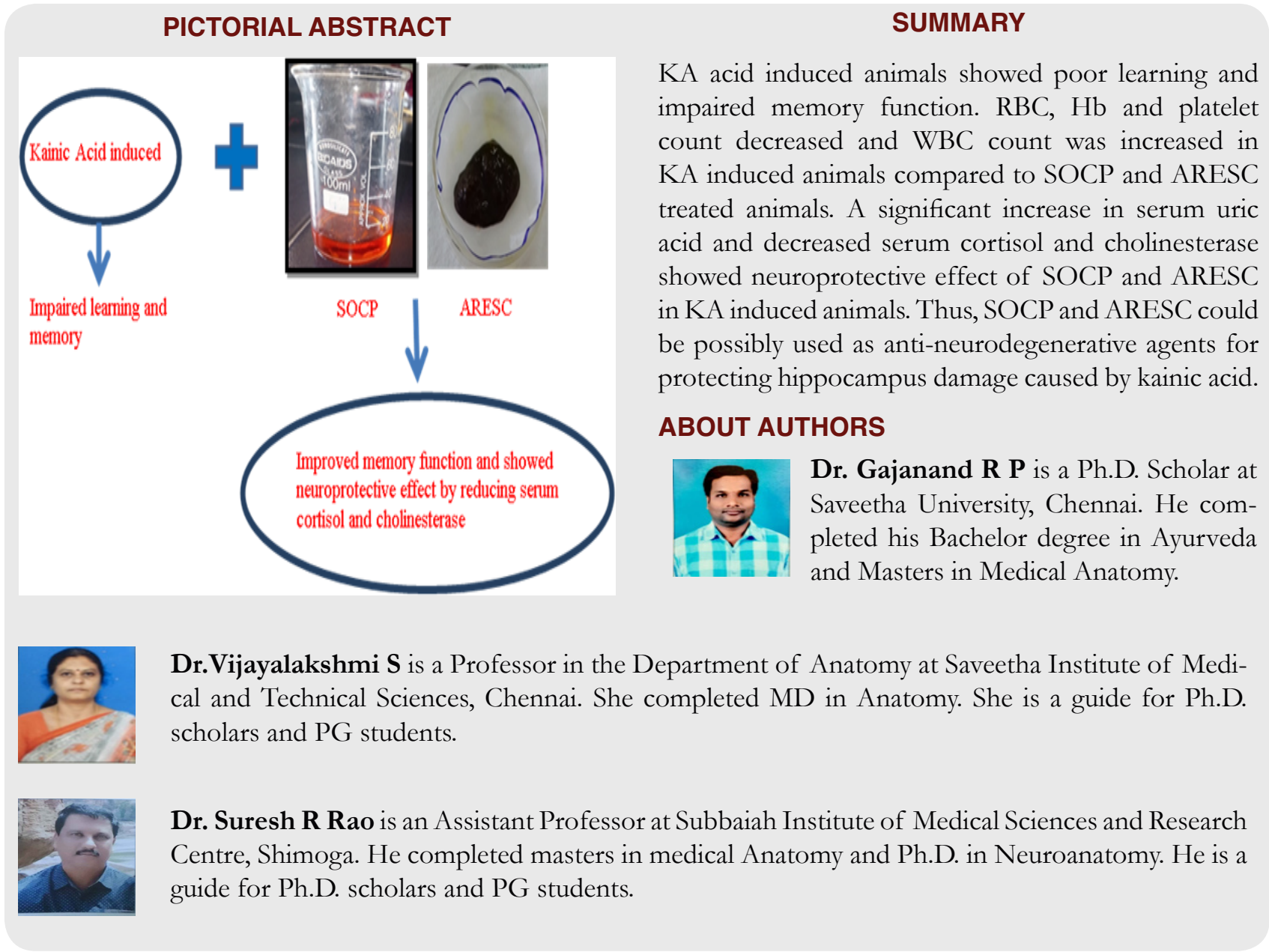

Cite this article: Gajanand RP, Vijayalakshmi S, Suresh R Rao. Effects of Celastrus paniculatus Willd. and Sida cordifolia Linn. in Kainic Acid Induced Hippocampus Damage in Rats. Indian J of Pharmaceutical Education and Research. 2019;53(3):537-44. 